\title{
Accurate calculation of resonances for a central-field model potential
}

\author{
Francisco M. Fernández \\ ‡ INIFTA (UNLP, CCT La Plata-CONICET), División Química Teórica, Blvd. 113 \\ S/N, Sucursal 4, Casilla de Correo 16, 1900 La Plata, Argentina \\ E-mail: fernande@quimica.unlp.edu.ar
}




\begin{abstract}
We obtain accurate resonance energies for the Schrödinger equation with a central-field potential by means of a method based on a rational approximation to the logarithmic derivative of the wavefunction. We discuss the rate of convergence of our approach and compare present results with those obtained earlier by other authors. We show that present method is superior to the spherical-box approach applied recently to the same problem. As far as we know present results are more accurate than those available in the literature and may be a suitable benchmark for testing future approaches.
\end{abstract}

\title{
1. Introduction
}

In a recent paper Zhou et al [1] applied the well-known spherical-box stabilization method to the calculation of the resonance energies of the Schrödinger equation with the potential $V(r)=V_{0} r^{2} e^{-r}+Z / r$. They integrated the eigenvalue equation by means of the Runge-Kutta method and estimated the positions and widths of the resonances from the behaviour of the bound-state energies as functions of the box radius. In particular they calculated the first two s-wave the first p-wave and the first d-wave resonances. Zhou et al [1] experienced some difficulties in estimating the position an width of the second s-wave resonance and could not obtain the third one. They also obtained rather crude estimations of the first p-wave and d-wave resonances. It seems that the spherical-box approach is rather ill-suited to broad resonances.

The potential $V(r)$ mentioned above has proved a suitable benchmark for the development and testing of several methods for the calculation of the energies of metastable states [2]24]. Most authors have considered the case $Z=0$ [2] 24] and just a few ones included the Coulomb interaction $Z=-1$ [20,23].

Some time ago, we applied the Riccati-Padé method (RPM) to the calculation of the lowest s-wave resonance of the potential $V(r)$ with $Z=0$ [17]. In that earlier paper we did not discuss the rate of convergence of the method on this particular model and merely showed the result for the lowest s-wave resonance. The purpose of this paper 
is to compare the RPM with the spherical-box approach and with other alternative methods on the central-field potential with $Z=-1$.

In Sec. 2 we outline the method. In Sec. 3 we apply the RPM to the potential $V(r)$ with $Z=-1$, analyze its results and compare them with those obtained earlier by other authors. Finally, in Sec. 4 we discuss the advantages of the RPM and draw conclusions.

\section{The method}

The radial part of the dimensionless Schrödinger equation for a central-field potential $V(r)$ is

$$
\left[-\frac{1}{2} \frac{d^{2}}{d r^{2}}+\frac{l(l+1)}{2 r^{2}}+V(r)\right] \Phi(r)=E \Phi(r)
$$

where $l=0,1, \ldots$ is the angular-momentum quantum number and $\Phi(0)=0$. The RPM is based on a rational approximation to the regularized logarithmic derivative of the wavefunction

$$
f(r)=\frac{l+1}{r}-\frac{\Phi^{\prime}(r)}{\Phi(r)}
$$

that can be expanded as follows:

$$
f(r)=\sum_{j=0}^{\infty} f_{j} r^{j}
$$

Note that the term $(l+1) / r$ removes the singularity of $\Phi^{\prime}(r) / \Phi(r)$ at origin and that we can obtain the coefficients $f_{j}(E)$ analytically by means of simple recurrence relations [17.

We then convert the Taylor series into a rational approximation:

$$
[N+d / N]=\frac{\sum_{j=0}^{N+d} a_{j} r^{j}}{\sum_{j=0}^{N} b_{j} r^{j}}=\sum_{j=0}^{2 N+d+1} f_{j} r^{j}
$$

where $N=1,2, \ldots$ and $d=0,1, \ldots$ The $2 N+d+1$ adjustable coefficients $a_{j}$ and $b_{j}$ are insufficient to provide the $2 N+d+2$ coefficients $f_{j}$. This condition is satisfied only if the Hankel determinant $H_{D}^{d}(E)$ with matrix elements $f_{i+j+d-1}(E), i, j=1,2, \ldots, D=N+1$, vanishes [17] (and references therein). The RPM conjecture is that there are sequences

of roots $E^{[D, d]}$ of $H_{D}^{d}(E)=0, D=2,3, \ldots$ that converge towards the actual bound- and 
metastable-state energies of the Schrödinger equation (11). The calculation is remarkably simple because the Hankel determinants are polynomial functions of the energy.

\section{Results and discussion}

For comparison we consider the potential

$$
V(r)=V_{0} r^{2} e^{-r}+\frac{Z}{r}
$$

with the model parameters $V_{0}=7.5$ and $Z=-1$ [1, 20, 23]. We label the complex energies of the metastable states $E_{l, \nu}$ so that $\operatorname{Re} E_{l, \nu+1}>\operatorname{Re} E_{l, \nu}, \nu=0,1, \ldots$ The s-, p-and d-waves discussed by Zhou et al [1] and Sofianos and Rakityansky [23] correspond to $l=0, l=1$, and $l=2$, respectively.

Since we are looking for Siegert pseudo states that satisfy [25]

$$
\lim _{r \rightarrow \infty} \frac{\Phi^{\prime}(r)}{\Phi(r)}=i k
$$

then it seems reasonable to choose $d=0$ because

$$
\lim _{r \rightarrow \infty}[N / N]=\frac{a_{N}}{b_{N}}
$$

For that reason it should be assumed that $d=0$ from now on, unless otherwise stated.

In order to estimate the rate of convergence of the RPM we calculate $L_{D}=$ $\log \left|\alpha_{D}-\alpha_{D+1}\right|$ where $\alpha_{D}$ is either the real or imaginary part of $E^{[D, 0]}$. Fig. 1] shows that the rate of convergence of the RPM for both the positions and widths of the first three $\mathrm{s}$-wave resonances is remarkable. It is worth mentioning that in the case of a narrow resonance the imaginary part of the root will appear at sufficiently large determinant dimensions $D$; that is to say, when $\left|\operatorname{Re} E^{[D, 0]}-\operatorname{Re} E^{[D+1,0]}\right|$ is of the order of magnitude of $|\operatorname{Im} E|$. We appreciate that the rate of convergence (given approximately by the slope of $L_{D}$ ) is almost independent of $\nu$; the main difference is that the greater the value of $\nu$ the larger the determinant dimension $D$ necessary for the appearance of the corresponding sequence. On the other hand, the performance of the spherical-box approach deteriorates as the resonance width increases [1. It is clear that the RPM is preferable to the spherical-box approach, at least for this example. 
Fig. 2 shows that the rate of convergence of the RPM for the p-wave resonances $(l=1)$ is similar to that discussed above. It is clear that the rate of convergence of the Hankel sequences is also independent of $l$. We confirm our conclusion that the RPM is preferable to the spherical-box approach because Zhou et al [1] roughly estimated the position and width of $E_{1,0}$ and were unable to obtain other p-wave resonances.

Fig. 3 shows the rate of convergence for the first two d-wave resonances. The behaviour is similar to those discussed above for the s and p ones. According to Zhou et al [1] the spherical-box approach only revealed the first $d$ resonance for which they could provide a rather crude estimate of the position and width.

As far as we know, the most accurate results for this model are those calculated some time ago by Sofianos and Rakityansky [23]. Present results are even more accurate and may therefore be a useful benchmark for other approaches. For that purpose we show them in Table 1.

Finally, we mention that the rate of convergence of the RPM is not affected by the choice of the displacement $d$. In the present case, for example, we obtained similar results with $d=1$ that we do not show here.

\section{Conclusions}

One of the main advantages of the RPM is its remarkable simplicity. We first obtain the coefficients of the Taylor series (3i) by means of a straightforward recurrence relation [17]. Second, we construct the Hankel determinant, which is a polynomial function of the energy, and find its roots. Third, we identify the sequences of roots that converge towards physically acceptable results.

Another advantage of the RPM is that exactly the same Hankel determinant applies to both the bound states and resonances. It comes from the fact that the RPM does not take explicitly into account the asymptotic form of the wavefunction at infinity and the rational approximation applies to any solution of the Schrödinger equation. Of course, we have to take into consideration the behaviour of the wavefunction at origin in order 
to remove any singularity of $\Phi^{\prime}(r) / \Phi(r)$.

We think that present results clearly show that the RPM is much more accurate and reliable than the spherical-box approximation. We have calculated the resonances discussed by Zhou et al [1] with much more accuracy and also obtained others that those authors were unable to locate. Besides, it is worth noting that the RPM is as simple, or even simpler, than the box-stabilization method in any of its forms [1,6, 13, 14,

[1] Zhou S-G, Meng J, and Zhao E-G 2009 J. Phys. B 42245001 (5 pp.).

[2] Bain R A, Bardsley J N, Junker B R, and Sukumar C V 1974 J. Phys. B 72189.

[3] Gazdy B 1976 J. Phys. A 9 L39.

[4] Isaacson A D, McCurdy C W, and Miller W H 1978 Chem. Phys. 34311.

[5] Junker B R 1980 Phys. Rev. Lett. 441487.

[6] Maier C H, Cederbaum L S, and Domcke W 1980 J. Phys. B 13 L119.

[7] Korsch H J, Laurent H, and Möhlenkamp R 1981 Molec. Phys. 431441.

[8] Junker B R, in 12th International Conference on the Physics of Electronic and Atomic Collisions, edited by S. Datz (North-Holland Publishing Company, Gatlinburg, Tennessee, 1982), p. 491.

[9] Korsch H J, Laurent H, and Möhlenkamp R 1982 J. Phys. B 151.

[10] Meyer H-D and Walter O 1982 J. Phys. B 153647.

[11] Connor J N L and Smith A D 1983 J. Chem. Phys. 786161.

[12] McCurdy C W, in Book Direct variational methods for complex resonance energies, edited by D. G. Truhlar (American Chemical Society, Washington, D. C., 1984), p. 17.

[13] Lefebvre R 1985 J. Phys. Chem. 894201.

[14] Mandelshtam V A, Ravuri T R, and Taylor H S 1993 Phys. Rev. Lett. 701932.

[15] Riss U V and Meyer H-D 1993 J. Phys. B 264503.

[16] Scrinzi A and Elander N 1993 J. Chem. Phys. 983866.

[17] Fernández F M 1995 J. Phys. A 284043.

[18] Lehr H and Chatzidimitriou-Dreismann C A 1995 Phys. Rev. A 513005.

[19] Riss U V and Meyer H-D 1995 J. Phys. B 281475.

[20] Yamani H A and Abdelmonem M S 1995 J. Phys. A 282709.

[21] Čižek M and Horáček J 1996 J. Phys. A 296325.

[22] Durand P and Paidarová I 1997 Czech. J. Phys. 47657.

[23] Sofianos S A and Rakityansky S A 1997 J. Phys. A 303725.

[24] Tolstikhin O I, Ostrovsky N, and Nakamura H 1998 Phys. Rev. A 582077.

[25] Siegert A J F 1939 Phys. Rev. 56750. 
Table 1. Some complex energies $E_{l, \nu}$ for the potential (5)

\begin{tabular}{llll}
\hline$l$ & $\nu$ & \multicolumn{1}{c}{$\operatorname{Re} E$} & \multicolumn{1}{c}{$\operatorname{Im} E=\Gamma / 2$} \\
\hline 0 & 0 & 1.7805245363623048 & 0.00004785969842876 \\
0 & 1 & 4.101494946209 & 0.578627213766 \\
0 & 2 & 4.6634610967 & 2.6832007703 \\
1 & 0 & 3.848001634811759 & 0.137692229585768 \\
1 & 1 & 4.750053489274 & 1.75278992436148 \\
2 & 0 & 4.9005161468291143 & 0.7837535082665858 \\
2 & 1 & 5.3006134902578 & 2.942357430621 \\
\hline
\end{tabular}

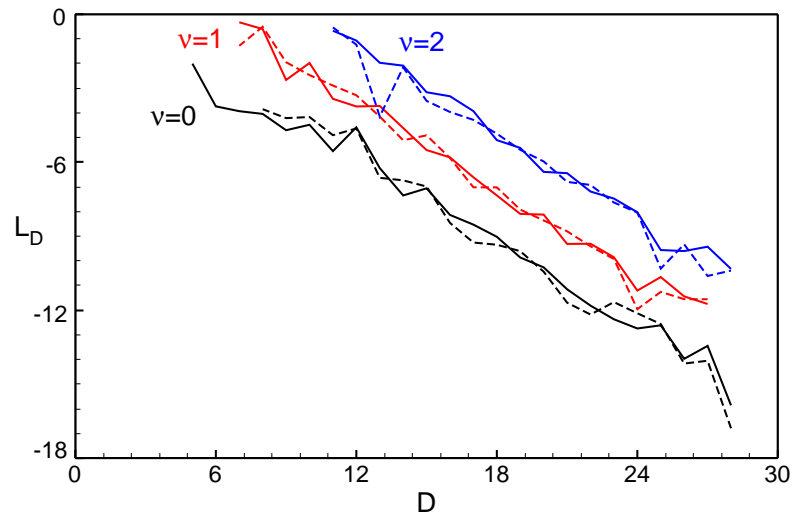

Figure 1. Convergence rate $L_{D}$ for the real (solid line) and imaginary (dashed line) parts of the energies $E_{0, \nu}$ 


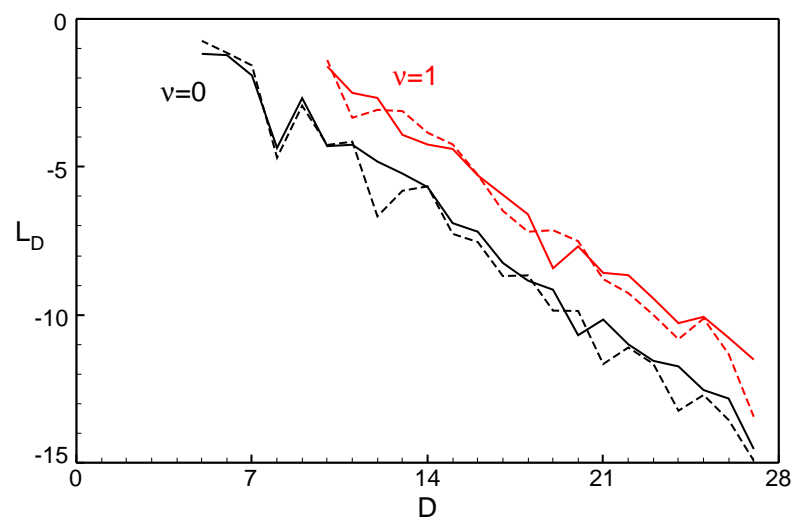

Figure 2. Convergence rate $L_{D}$ for the real (solid line) and imaginary (dashed line) parts of the energies $E_{1, \nu}$

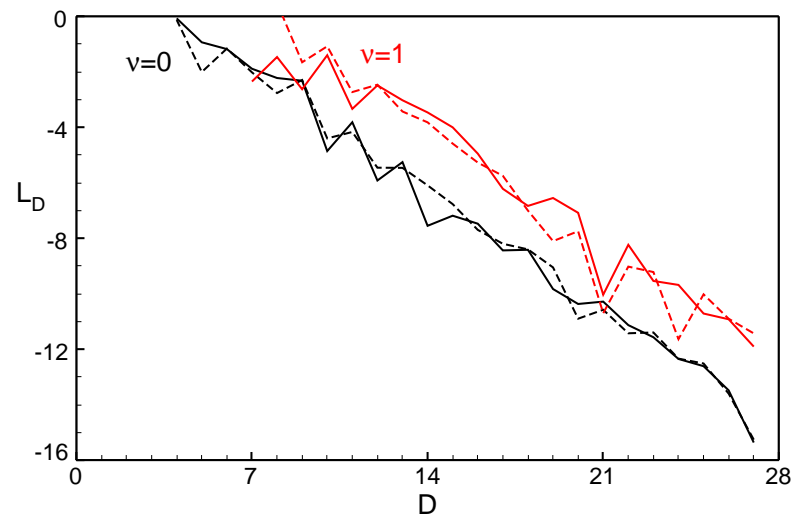

Figure 3. Convergence rate $L_{D}$ for the real (solid line) and imaginary (dashed line) parts of the energies $E_{2, \nu}$ 